\title{
Non-Alcoholic Fatty Liver and Metabolic Syndrome in Children: A Vicious Circle
}

\author{
Arianna Alterio Anna Alisi Daniela Liccardo Valerio Nobili \\ Hepato-Metabolic Disease Unit and Liver Research Unit, 'Bambino Gesù' Children's Hospital, IRCCS, Rome, Italy
}

\section{Key Words}

Non-alcoholic fatty liver disease $\cdot$ Metabolic syndrome . Obesity $\cdot$ Insulin resistance $\cdot$ Children

\begin{abstract}
During the last decade, paediatricians have observed a dramatic increase of non-alcoholic fatty liver disease (NAFLD) and metabolic syndrome (MS) in children. Furthermore, several lines of evidence have reported that a large part of children with NAFLD presents one or more traits of MS making plausible that, in the coming years, these subjects may present a rapid course of disease towards more severe cirrhosis and cardiovascular disease. Genetic susceptibility and the pressure of intrauterine environment and lifestyle are all crucial to activate molecular machinery that leads to development of NAFLD and MS in childhood. In this scenario, central obesity and consequent adipose tissue inflammation are critical to promote both MS-associated metabolic dysfunctions and NAFLD-related hepatic damage. An excessive dietary intake may in fact cause a specific lipid partitioning and induce metabolic stressors, which in turn promote insulin resistance and the release of several circulating factors. These molecules, on the one hand, trigger steatosis and the inflammatory response that characterize liver damage in NAFLD, and on the other hand contribute to the onset of other features of MS. This review provides an overview of current genetic, pathogenetic and clinical evidence of the vicious circle created by NAFLD and MS in children.
\end{abstract}

(c) 2014 S. Karger AG, Basel

\section{KARGER}

(c) 2014 S. Karger AG, Basel

$1663-2818 / 14 / 0825-0283 \$ 39.50 / 0$

E-Mail karger@karger.com

www.karger.com/hrp

\section{Introduction}

Non-alcoholic fatty liver disease (NAFLD) is the most common cause of chronic liver disease in Western countries and its worldwide prevalence in adults and children is increasing and becoming an obesity epidemic $[1,2]$. Particularly, there is an alarming increase of number of children affected by NAFLD supported by high prevalence data ranging from 3 to $12 \%$ in the general paediatric population up to $70-90 \%$ in young obese [3].

Paediatric NAFLD is associated with several factors of metabolic syndrome (MS), like abdominal (central) obesity, dyslipidaemia (hypertriglyceridaemia and/or hypercholesterolaemia) and insulin resistance. Therefore, NAFLD can be considered as the hepatic manifestation of MS, even if there is no agreement that NAFLD contributes to MS and vice versa [4]. In fact, the aetiology and pathogenesis of both diseases are multifaceted and closely related to genetic predisposing factors, intrauterine environment and unhealthy lifestyle [5]. Bad eating habits and sedentarity may favour the onset of childhood obesity strongly contributing to make a child more prone to develop NAFLD and MS during adolescence [6]. Furthermore, as a large part of children with NAFLD and traits of MS remain undiagnosed, it is plausible that, in the coming years, adolescents with NAFLD characteristics may present a rapid course of disease towards more severe forms of liver damage (i.e. cirrhosis), MS and associated cardiovascular disease (CVD). In fact, during the 
last decade, several epidemiological data have revealed a dramatic increase of the MS incidence in urban adolescents mainly due to overnutrition and a sedentary lifestyle [7].

In this scenario, the role of unhealthy lifestyle prevention is critical, particularly in subjects genetically predisposed to both diseases. Here, we provide an overview of current genetic, pathogenetic and clinical evidence of the vicious circle created by NAFLD and MS in children.

\section{Methods}

A PubMed/MEDLINE search of the literature in the last 5 years was performed, using the following search terms: NAFLD and MS and children. This search yielded 121 publications of which 35 were relevant recent clinical studies and 6 were review articles. To these we added 12 relevant articles on NAFLD and MS published between 2009 and 2013.

\section{Characteristics That Define NAFLD and MS in Children}

\section{Non-Alcoholic Fatty Liver Disease}

NAFLD is defined as an intrahepatic fat accumulation in people who drink little or no alcohol ( $>20 \mathrm{~g} /$ day). In general, it is considered a multifactorial liver disease that ranges from benign steatosis to the more severe non-alcoholic steatohepatitis (NASH), which is characterized in children by lobular inflammation and hepatocellular ballooning, and frequently coupled to fibrosis $[2,8]$.

During NAFLD pathogenesis, according to the 'multiple hits' hypothesis, two primary hits including insulin resistance and free fatty acid (FFA) accumulation induce steatosis ( $>5 \%$ of hepatocytes, histologically) making the liver more susceptible to secondary hits, such as the action of adipocytokines, oxidative stressors and immune response that subsequently lead to NASH and fibrogenesis [9]. It is well recognized that diet, exercise, central obesity, insulin resistance, and hyperlipidaemia strongly influence the epigenetic dependent development and progression of NAFLD as well as MS. However, not all patients with this pedigree of risk factors for MS develop NAFLD. Therefore, it is not surprising that a possible genetically established predisposition, including single nucleotide polymorphisms (SNPs), may explain both the ethnic differences in the prevalence of disease and an increased risk for advanced NASH and fibrosis [2].

\section{Metabolic Syndrome}

MS in adults is defined by the International Diabetes Federation (IDF) as the presence of abdominal obesity (by measuring waist circumference) and two or more of the following factors: hypertriglyceridaemia, low HDL cholesterol, fasting glucose and hypertension (http://www. idf.org/idf-worldwide-definition-metabolic-syndrome). IDF proposed similar criteria for definition of MS in the paediatric population according to three age groups: from 6 to 10 years, from 10 to 16 years, and $\geq 16$ years was considered as adults (http://www.idf.org/node/1405? unode= 4A7F23CB-FA35-4471-BB06-0294AD33F2FC) [9].

The pathogenesis of MS is not completely understood but two factors are certainly crucial in its genesis: central obesity and insulin resistance. Central obesity is associated with hypertension, hypertriglyceridaemia and hyperglycaemia. Children with excess fat have a higher risk of CVD in adulthood [10]. In fact, when white adipose tissue (WAT) excess is localized in the upper parts of the body, defining central obesity, it represents a strong risk factor for metabolic and pro-inflammatory complications [11]. Therefore, even in the case of MS a genetic susceptibility to WAT accumulation concomitantly to lifestyle factors (i.e. diet and exercise) may lead to a specific lipid partitioning and consequent adipose tissue inflammation and increased FFA flux, which drive the various elements of disease including insulin resistance and affect endothelial function [12].

\section{Role of Genetic Predisposition}

A large part of the translational research is today devoted to the pursuit of genetic variants that can be used to identify subjects with a genetic susceptibility or resistance to multifactorial diseases, including NAFLD and MS. These genetic variants in the DNA code (known as alleles), in hetero- or homozygosity, may be associated with specific traits of these diseases acquiring a high predictive value as markers. Variants at a single DNA basepair or SNPs have received a great attention as potential genetic markers. They have the advantage of a high frequency in the human genome (on average, 1 occurs in every 1,000 nucleotides) and are relatively easy to genotype using next-generation sequencing technologies. Although genetic susceptibility to NAFLD and MS are not completely known, it was suggested that their development may be associated with SNPs in genes involved in the control of insulin resistance, lipid metabolism, inflammation and oxidative stress [5]. 
Fig. 1. Schematic summary of the hypothetical mechanisms that sustain the vicious circle between NAFLD and MS in children.

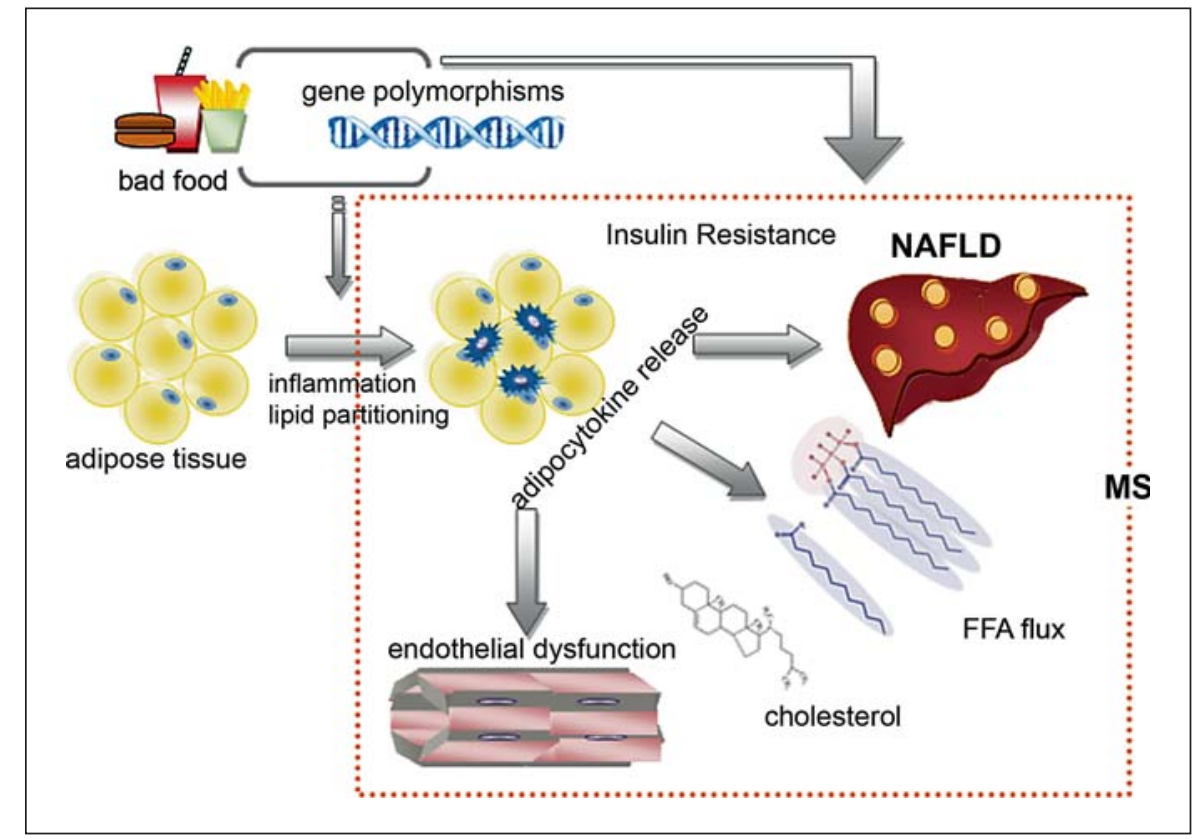

During last 5 years, several potential genetic SNPs have been suggested as potential candidates for either paediatric NAFLD susceptibility or disease progression (i.e. NASH and fibrosis), including the rs738409 variant of adiponutrin/patatin-like phospholipase domain-containing 3 (PNPLA3) coding gene, the rs 1801278 variant of insulin receptor substrate-1 (IRS-1) coding gene, the rs3750861 variant of Kruppel-like factor 6 (KLF6) gene and the rs4880 variant of manganese-dependent superoxide dismutase (SOD2) gene. Genome-wide association studies have identified many SNPs associated with a large number of conditions related to MS [13]. However, only recently it has been reported that the rs 1800849 variant of uncoupling protein 3 (UCP3) gene associates with MS components and increased risk of Chinese children with NAFLD [14]. Moreover, as the severity of obstructive sleep apnoea (OSAS) also correlates with the increased risk of paediatric NAFLD and MS, the presence of selective SNPs in the gene encoding for fatty acid-binding protein 4 (FABP4) might explain the susceptibility to OSAS in both diseases $[15,16]$.

\section{Role of Central Obesity-Dependent Inflammation and Insulin Resistance}

Central obesity correlates with an accumulation of visceral fat, which in turn depends on adipose tissue dysfunctions. In fact, adipose tissue is now considered like an 'endocrine' organ, that may produce and release circulating factors, called adipocytokines, that are involved in the alterations of MS. In fact, in adipocytes, bad dietary intake (i.e. elevated consumption of sweet high-fat foods) causes a specific pattern of lipid storage and metabolic stress, which in turn activate signalling cascades that induce oxidative stress and trigger an inflammatory response. Therefore, metabolic stressors and lipid partitioning may result in the release of several circulating adipocytokines with local action (i.e. on macrophages), and systemic effects such as muscle and liver insulin resistance. Furthermore, these circulating factors including tumour necrosis factor $\alpha$ (TNF- $\alpha$ ), interleukin 6 (IL-6), leptin, adiponectin, retinol-binding protein-4 (RBP4) and resistin play a critical role in inflammation that often characterized subjects with insulin resistance, NAFLD and MS predisposition $[5,17,18]$. This nexus between NAFLD and MS, summarized in figure 1 , is supported by clinical evidence reported in children.

\section{Evidence of NAFLD and MS Nexus in Children}

Over the last decade there has been increased interest in the determination of possible pathogenetic and clinical relationships between NAFLD and MS in obese subjects during childhood $[5,19]$. A retrospective study including 43 American children with biopsy-proven NAFLD has provided the first evidence of NAFLD/MS association at paediatric age reporting that approximately $95 \%$ of patients with liver disease were obese and were insulin-re- 
sistant [20]. Accordingly, Manco et al. [21] have demonstrated that 92 and $84 \%$ of 197 Caucasian children with NAFLD displayed a body mass index $(\mathrm{BMI})>85$ th percentile and waist circumference $\geq 90$ th percentile, respectively. The strong correlation between central obesity and elevated waist circumferences has also been confirmed by a case-control study conducted on 150 overweight/obese children with biopsy-proven NAFLD and 150 without [22]. Moreover, the same authors found that among the subjects studied, MS showed 5.0 times the odds of having NAFLD compared to without the metabolic disease.

Further cross-sectional studies conducted on different ethnic groups have supported the connection between NAFLD and MS in the paediatric population. Kelishadi et al. [23] have in fact reported that in 1,107 Iranian subjects (aged 6-18 years) central obesity may be used as a predictor of NAFLD assessed by ultrasound and surrogate markers, such as alanine aminotransferase (ALT). Accordingly, Wei et al. [24] have demonstrated that approximately $16 \%$ of a cohort of obese British children display an increase of serum ALT levels, elevated BMI and altered glucose homeostasis. It has also been reported that in Chinese obese children the overlapping between NAFLD and MS may be found in $84.61 \%$ of cases [25]. Interestingly, Deboer et al. [26] have found that ALT elevation varied by race/ethnicity demonstrating that nonHispanic Black adolescents exhibit a relationship between insulin resistance and ALT lower than in non-Hispanic White and Hispanic age-matched subjects, supporting the weight of genetic predisposition in the link between NAFLD and MS. More recently, two other prospective studies have confirmed a positive association between elevated ALT levels and MS prevalence in overweight and obese Hispanic children observed in tertiary care centres $[27,28]$. Finally, very recently a cross-sectional study on 182 obese sedentary children and adolescents (6-16 years) has documented the significant correlation between intra-abdominal fat and NAFLD $(p=0.005)$, MS $(\mathrm{p}=0.013)$, dyslipidaemia $(\mathrm{p}=0.001)$ and HOMA-IR $(\mathrm{p}=0.007)[29]$.

Besides central obesity, adipocytokine circulating levels have also been demonstrated as a common feature between NAFLD and MS in children. Interestingly, among these adipocytokines, leptin serum levels increase in concert to steatosis grade, ballooning, inflammation and fibrosis severity, indicating that hyperleptinaemia in NAFLD children could also be a prerequisite for insulin resistance and other MS features in these subjects [29]. On the contrary, adiponectin and resistin act as anti-inflammatory molecules, because their circulating levels display a negative correlation with liver steatosis assessed by abdominal ultrasound [30]. Moreover, Lebensztejn et al. [31] have clarified that only hypoadiponectinaemia is significantly associated with a decreased risk of NAFLD and insulin resistance. As adiponectin and leptin may control the expression and secretion of pro-inflammatory cytokines such as TNF- $\alpha$ and IL-6, it is expected that these adipose tissue-dependent circulating molecules also associate with NAFLD and insulin resistance [32]. The real role of these two adipocytokines in the development and progression of NAFLD in children remains to be defined. However, Mager et al. [33] reported that meals high in saturated fat may lead to postprandial dyslipidaemia and hyperinsulinaemia concomitantly to a change in pro-inflammatory and lipoprotein pattern in obese children with and without NAFLD.

In addition to these commonly studied adipocytokines, the circulating levels of RBP4, which are strongly associated with insulin resistance, also display an inverse association with liver damage progression in paediatric NAFLD [34]. On the contrary, Boyraz et al. [35] have recently demonstrated that RBP4 levels are increased in 63 obese children with ultrasound and ALT elevation evidence of NAFLD.

Although several differences exist in the power of correlation of these adipocytokine levels with the severity of NAFLD and MS traits, recent clinical evidence reinforces the role of these circulating molecules as biomarkers of both diseases [36-38]. Noteworthy, Walker et al. [39] have recently suggested the connection between WAT inflammation, adipocytokine levels and NAFLD severity in children, demonstrating that the increasing obesity is, on the one hand, strongly related to increased intrahepatic lipid deposition and adipocytokine-dependent activation of Kupffer cells explaining the progression from NAFLD to NASH, and, on the other hand, it is also significantly associated with markers of adipose tissue damage via crown-like structure resembling the process of liver fibrogenesis.

\section{Role of Intrauterine Environment in NAFLD and MS Cross-Talk}

Intrauterine growth retardation (IUGR) is the most important cause of perinatal mortality and morbidity, and is often used as a synonym of small for gestational age (SGA). Interestingly, several studies have highlighted the relationship between IUGR and the increased risk of MS in children [5]. The pathogenetic machinery that pro- 
motes MS in IUGR remains unclear, even though the increase of insulin resistance appears to play a pivotal role by two potential mechanisms: (1) the establishment of an insulin-resistant genotype independently of intrauterine environment, and (2) the foetal reprogramming due to a thrifty phenotype hypothesis [40]. Furthermore, experimental studies have demonstrated that IUGR is associated with not only obesity and lipid abnormalities, but also with liver steatosis and inflammation [40]. Interestingly, Nobili et al. [41] have found in 90 Italian children an association between paediatric NAFLD with IUGR, independently of insulin resistance. Accordingly, Faienza et al. [42] more recently have reported $34.8 \%$ of NAFLD out of 23 SGA children demonstrating that the insulin resistance index significantly correlates with liver steatosis at ultrasound.

Although significant gaps still exist in the mechanisms inducing metabolic profile abnormalities and NAFLD in children, both diseases should be recognized as a coupled emerging health problem in IUGR/SGA prepubertal individuals.

\section{Emerging Role of Hepatokines and Thyroid Hormones}

Liver responds to adipose tissue inflammation not only with the histological damage that characterizes NAFLD but also with production and release of some circulating molecules called hepatokines, which may directly promote metabolic dysfunctions that lead to MS [43]. In particular, three hepatokines, including insulin-like growth factors (IGF)-I and -II, fibroblast growth factor 21 (FGF21) and fetuin-A, have been reported as novel promising therapeutic targets and non-invasive biomarkers for paediatric NAFLD and MS. Interestingly, Cianfarani et al. [44] have recently demonstrated that obese children with NAFLD display decreased levels of IGF-I and -II that correlate both with insulin resistance and severity of liver disease. Moreover, it has been reported that circulating levels of FGF21 are not only increased in obese children but, more importantly, there is an inverse correlation between the serum levels of this hepatokine and paediatric NAFLD-related liver tissue damage [45]. Finally, Reinehr et al. [46] demonstrated that serum levels of fetuin-A, an inhibitor of tyrosine kinase activity of the insulin receptor in the liver, are elevated in NAFLD children and correlate with insulin resistance.

Interestingly, some previous studies have addressed the association between thyroid dysfunction and NAFLD, supporting a pathogenetically plausible explanation given by the association between the common features of MS observed in both NAFLD and hypothyroidism [47]. Pacifico et al. [48] provide the first evidence between NAFLD, thyroid function and MS in childhood. These authors have demonstrated that thyroid function tests, particularly the thyroid-stimulating hormone (TSH) test, positively associate with NAFLD and metabolic variables in 402 consecutive overweight/obese children, suggesting TSH as a potential predictor of liver disease and lipid and glucose dysmetabolism, independently of visceral obesity. Accordingly, Torun et al. [49] have reported that free thyroxine and free triiodothyronine levels are comparable in obese children and age- and gender-matched subjects with normal weight, whereas TSH levels significantly increase correspondingly to the steatosis degree assessed by ultrasound, and correlate with ALT and BMI.

\section{NAFLD and MS Collaboration in Early CVD Development}

The concept that NAFLD and MS may represent overlapping diseases is also suggested by the fact that intrahepatic fat accumulation seems to predict more strongly than central obesity the likelihood that metabolic abnormalities are associated with early CVD in youth [10]. In fact, as demonstrated by several authors, children with NAFLD may present certain endothelial dysfunctions and greater carotid intima-media thickness, the latter considered a surrogate marker of early atherogenesis in childhood [50-53]. In fact, Nobili et al. [50] have shown for the first time that in children with NAFLD, the severity of liver injury is strongly associated with the presence of a more atherogenic lipid profile measured as triglyceride/high-density lipoprotein cholesterol (HDL), total cholesterol/HDL, and low-density lipoprotein cholesterol (LDL)/HDL ratios. Accordingly, Pacifico et al. [51] have demonstrated in a population study including 548 children that subjects with a high triglycerides/HDL-C ratio have an increased risk of insulin resistance and display an association between this ratio and NAFLD, independently of obesity.

Left ventricular (LV) dysfunction is a further abnormality in cardiac function that has recently been found to be associated with NAFLD and MS in children. In particular, Fintini et al. [52] have demonstrated that LV hypertrophy can be detectable early in NAFLD children even if it does not appear to be linked to other cardiovascular and metabolic alterations. Moreover, Pacifico et al. 
[53] have found that asymptomatic obese children with NAFLD exhibit early LV diastolic and systolic dysfunctions that become more severe in NASH subjects.

\section{Conclusions and Future Perspectives}

The rapid prevalence of NAFLD and MS among children in recent years has made research urgent to gain the scientific knowledge required of genetic and molecular mechanisms in order to perform possibly early prevention and design efficient therapeutic approaches. Several lines of clinical evidence have demonstrated that a vicious circle exists between NAFLD and MS in children. It is plausible that a common genetic predisposition may provide fertile soil for the complex molecular overlap of multiorgan response to different intrauterine and postnatal environmental factors, even though much remains to be explored in this field. Furthermore, as NAFLD and MS phenotypes are associated with a high risk of CVD, such information may be critical for the development of public health promotion strategies that approach children as future adults. In the meantime, as the adipose tissue-dependent inflammation and its cross-talk with the liver appears to be in some cases central for the NAFLD/MS connection, weight loss and weight control, coupled with a multidisciplinary approach to metabolic dysfunction and liver damage, should be considered by paediatricians the first-line treatment in the management of both diseases.

\section{References}

1 Vernon G, Baranova A, Younossi ZM: Systematic review: the epidemiology and natural history of non-alcoholic fatty liver disease and non-alcoholic steatohepatitis in adults. Aliment Pharmacol Ther 2011;34:274-285.

-2 Nobili V, Svegliati-Baroni G, Alisi A, Miele L, Valenti L, Vajro P: A 360-degree overview of paediatric NAFLD: recent insights. J Hepatol 2013;58:1218-1229.

3 Alisi A, Manco M, Vania A, Nobili V: Pediatric nonalcoholic fatty liver disease in 2009. J Pediatr 2009;155:469-474.

-4 Alisi A, Feldstein AE, Villani A, Raponi M, Nobili V: Pediatric nonalcoholic fatty liver disease: a multidisciplinary approach. Nat Rev Gastroenterol Hepatol 2012;9:152-161.

5 Alisi A, Cianfarani S, Manco M, Agostoni C, Nobili V: Non-alcoholic fatty liver disease and metabolic syndrome in adolescents: pathogenetic role of genetic background and intrauterine environment. Ann Med 2012;44: $29-40$.

6 Edmison JM, Kalhan SC, McCullough AJ: Obesity, hepatic metabolism and disease. Nestle Nutr Workshop Ser Pediatr Program 2009;63:163-172.

7 Steinberger J, Daniels SR, Eckel RH, Hayman L, Lustig RH, McCrindle B, Mietus-Snyder ML; American Heart Association Atherosclerosis, Hypertension, and Obesity in the Young Committee of the Council on Cardiovascular Disease in the Young; Council on Cardiovascular Nursing; and Council on $\mathrm{Nu}$ trition, Physical Activity, and Metabolism: Progress and challenges in metabolic syndrome in children and adolescents: a scientific statement from the American Heart Association Atherosclerosis, Hypertension, and Obesity in the Young Committee of the Council on Cardiovascular Disease in the Young; Council on Cardiovascular Nursing; and Council on Nutrition, Physical Activity, and Metabolism. Circulation 2009;119:628647.

8 Brunt EM: Pathology of nonalcoholic fatty liver disease. Nat Rev Gastroenterol Hepatol 2010;7:195-203.

-9 Alberti KG, Zimmet P, Shaw J; IDF Epidemiology Task Force Consensus Group: The metabolic syndrome - a new worldwide definition. Lancet 2005;366:1059-1062.

10 Pacifico L, Nobili V, Anania C, Verdecchia P, Chiesa C: Pediatric nonalcoholic fatty liver disease, metabolic syndrome and cardiovascular risk. World J Gastroenterol 2011;17: 3082-3091.

11 Balistreri CR, Caruso C, Candore G: The role of adipose tissue and adipokines in obesityrelated inflammatory diseases. Mediators Inflamm 2010;2010:802078.

12 Weiss R, Bremer AA, Lustig RH: What is metabolic syndrome, and why are children getting it? Ann NY Acad Sci 2013;1281:123-140.

13 Pollex RL, Hegele RA: Genetic determinants of the metabolic syndrome. Nat Clin Pract Cardiovasc Med 2006;3:482-489.

14 Xu YP, Liang L, Wang CL, Fu JF, Liu PN, Lv LQ, Zhu YM: Association between UCP3 gene polymorphisms and nonalcoholic fatty liver disease in Chinese children. World J Gastroenterol 2013;19:5897-5903.

15 Nobili V, Cutrera R, Liccardo D, Pavone M, Devito R, Giorgio V, Verrillo E, Baviera G, Musso G: Obstructive sleep apnea syndrome affects liver histology and inflammatory cell activation in pediatric nonalcoholic fatty liver disease, regardless of obesity/insulin resistance. Am J Respir Crit Care Med 2014;189: 66-76.

16 Bhushan B, Khalyfa A, Spruyt K, Kheirandish-Gozal L, Capdevila OS, Bhattacharjee R, Kim J, Keating B, Hakonarson H, Gozal D:
Fatty-acid binding protein 4 gene polymorphisms and plasma levels in children with obstructive sleep apnea. Sleep Med 2011;12: 666-671.

17 Wozniak SE, Gee LL, Wachtel MS, Frezza EE: Adipose tissue: the new endocrine organ? A review article. Dig Dis Sci 2009;54:18471856.

18 Asrih M, Jornayvaz FR: Inflammation as a potential link between nonalcoholic fatty liver disease and insulin resistance. J Endocrinol 2013;218:R25-R36.

19 Nobili V, Bedogni G, Berni Canani R, Brambilla P, Cianfarani S, Pietrobelli A, Agostoni $\mathrm{C}$ : The potential role of fatty liver in paediatric metabolic syndrome: a distinct phenotype with high metabolic risk? Pediatr Obes 2012; 7:e75-e80.

20 Schwimmer JB, Deutsch R, Rauch JB, Behling C, Newbury R, Lavine JE: Obesity, insulin resistance, and other clinicopathological correlates of pediatric nonalcoholic fatty liver disease. J Pediatr 2003;143:500-505.

21 Manco M, Bedogni G, Marcellini M, Devito R, Ciampalini P, Sartorelli MR, Comparcola D, Piemonte F, Nobili V: Waist circumference correlates with liver fibrosis in children with non-alcoholic steatohepatitis. Gut 2008; 57:1283-1287.

22 Schwimmer JB, Pardee PE, Lavine JE, Blumkin AK, Cook S: Cardiovascular risk factors and the metabolic syndrome in pediatric nonalcoholic fatty liver disease. Circulation 2008; 118:277-283

23 Kelishadi R, Cook SR, Adibi A, Faghihimani Z, Ghatrehsamani S, Beihaghi A, Salehi H, Khavarian N, Poursafa P: Association of the components of the metabolic syndrome with nonalcoholic fatty liver disease among normalweight, overweight and obese children and adolescents. Diabetol Metab Syndr 2009;1:29. 
-24 Wei C, Ford A, Hunt L, Crowne EC, Shield JP: Abnormal liver function in children with metabolic syndrome from a UK-based obesity clinic. Arch Dis Child 2011;96:1003-1007.

-25 Fu JF, Shi HB, Liu LR, Jiang P, Liang L, Wang CL, Liu XY: Non-alcoholic fatty liver disease: an early mediator predicting metabolic syndrome in obese children? World J Gastroenterol 2011;17:735-742.

-26 Deboer MD, Wiener RC, Barnes BH, Gurka MJ: Ethnic differences in the link between insulin resistance and elevated ALT. Pediatrics 2013;132:e718-e726.

-27 Engelmann G, Hoffmann GF, Grulich-Henn J, Teufel U: Alanine aminotransferase elevation in obese infants and children: a marker of early onset non-alcoholic fatty liver disease. Hepat Mon 2014;14:e14112.

28 Elizondo-Montemayor L, Ugalde-Casas PA, Lam-Franco L, Bustamante-Careaga H, Serrano-González M, Gutiérrez NG, Martínez U: Association of ALT and the metabolic syndrome among Mexican children. Obes Res Clin Pract 2014;8:e79-e87.

-29 Silveira LS, Monteiro PA, Antunes Bde M, Seraphim PM, Fernandes RA, Christofaro DG, Freitas Júnior IF: Intra-abdominal fat is related to metabolic syndrome and non-alcoholic fat liver disease in obese youth. BMC Pediatr 2013;13:115.

-30 Nobili V, Manco M, Ciampalini P, Diciommo V, Devito R, Piemonte F, Comparcola D, Guidi R, Marcellini M: Leptin, free leptin index, insulin resistance and liver fibrosis in children with non-alcoholic fatty liver disease. Eur J Endocrinol 2006;155:735-743.

- 31 Lebensztejn DM, Wojtkowska M, Skiba E, Werpachowska I, Tobolczyk J, Kaczmarski M: Serum concentration of adiponectin, leptin and resistin in obese children with nonalcoholic fatty liver disease. Adv Med Sci 2009;54:177-182.

- 32 Tilg H, Hotamisligil GS: Nonalcoholic fatty liver disease: cytokine-adipokine interplay and regulation of insulin resistance. Gastroenterology 2006;131:934-945.

- 33 Mager DR, Mazurak V, Rodriguez-Dimitrescu C, Vine D, Jetha M, Ball G, Yap J: A meal high in saturated fat evokes postprandial dyslipemia, hyperinsulinemia, and altered lipoprotein expression in obese children with and without nonalcoholic fatty liver disease. JPEN J Parenter Enteral Nutr 2013;37:517-528.
34 Nobili V, Alkhouri N, Alisi A, Ottino S, Lopez R, Manco M, Feldstein AE: Retinol-binding protein 4: a promising circulating marker of liver damage in pediatric nonalcoholic fatty liver disease. Clin Gastroenterol Hepatol 2009; 7:575-579.

35 Boyraz M, Cekmez F, Karaoglu A, Cinaz P, Durak M, Bideci A: Serum adiponectin, leptin, resistin and RBP4 levels in obese and metabolic syndrome children with nonalcoholic fatty liver disease. Biomark Med 2013;7: 737-745.

-36 Fitzpatrick E, Dew TK, Quaglia A, Sherwood RA, Mitry RR, Dhawan A: Analysis of adipokine concentrations in paediatric non-alcoholic fatty liver disease. Pediatr Obes 2012;7: 471-479.

- 37 Kim JS, Lê KA, Mahurkar S, Davis JN, Goran MI: Influence of elevated liver fat on circulating adipocytokines and insulin resistance in obese Hispanic adolescents. Pediatr Obes 2012;7:158-164.

38 Koot BG, van der Baan-Slootweg OH, Bohte AE, Nederveen AJ, van Werven JR, Tamminga-Smeulders CL, Merkus MP, Schaap FG, Jansen PL, Stoker J, Benninga MA: Accuracy of prediction scores and novel biomarkers for predicting non-alcoholic fatty liver disease in obese children. Obesity (Silver Spring) 2013; 21:583-590.

39 Walker RW, Allayee H, Inserra A, Fruhwirth R, Alisi A, Devito R, Carey ME, Sinatra F, Goran MI, Nobili V: Macrophage accumulation and fibrosis in adipose tissue is linked to liver damage and metabolic risk in obese children. Obesity (Silver Spring) 2014;22:1512-1519.

40 Alisi A, Panera N, Agostoni C, Nobili V: Intrauterine growth retardation and nonalcoholic fatty liver disease in children. Int J Endocrinol 2011:2011:269853.

41 Nobili V, Marcellini M, Marchesini G, Vanni E, Manco M, Villani A, Bugianesi E: Intrauterine growth retardation, insulin resistance, and nonalcoholic fatty liver disease in children. Diabetes Care 2007;30:2638-2640.

-42 Faienza MF, Brunetti G, Ventura A, D’Aniello M, Pepe T, Giordano P, Monteduro M, Cavallo L: Nonalcoholic fatty liver disease in prepubertal children born small for gestational age: influence of rapid weight catch-up growth. Horm Res Paediatr 2013;79:103-109.

43 Stefan N, Häring HU: The role of hepatokines in metabolism. Nat Rev Endocrinol 2013;9: 144-152.
44 Cianfarani S, Inzaghi E, Alisi A, Germani D, Puglianiello A, Nobili V: Insulin-like growth factor-I and -II levels are associated with the progression of nonalcoholic fatty liver disease in obese children. J Pediatr 2014;165: 92-98.

45 Alisi A, Ceccarelli S, Panera N, Prono F, Petrini S, De Stefanis C, Pezzullo M, Tozzi A, Villani A, Bedogni G, Nobili V: Association between serum atypical fibroblast growth factors 21 and 19 and pediatric nonalcoholic fatty liver disease. PLoS One 2013;8:e67160.

46 Reinehr T, Roth CL: Fetuin-A and its relation to metabolic syndrome and fatty liver disease in obese children before and after weight loss. J Clin Endocrinol Metab 2008;93:4479-4485.

47 Chung GE, Kim D, Kim W, Yim JY, Park MJ, Kim YJ, Yoon JH, Lee HS: Non-alcoholic fatty liver disease across the spectrum of hypothyroidism. J Hepatol 2012;57:150-156.

48 Pacifico L, Bonci E, Ferraro F, Andreoli G Bascetta S, Chiesa C: Hepatic steatosis and thyroid function tests in overweight and obese children. Int J Endocrinol 2013;2013: 381014

49 Torun E, Özgen IT, Gökçe S, Aydın S, Cesur Y: Thyroid hormone levels in obese children and adolescents with non-alcoholic fatty liver disease. J Clin Res Pediatr Endocrinol 2014;6: 34-39.

50 Nobili V, Alkhouri N, Bartuli A, Manco M, Lopez R, Alisi A, Feldstein AE: Severity of liver injury and atherogenic lipid profile in children with nonalcoholic fatty liver disease. Pediatr Res 2010;67:665-670.

-51 Pacifico L, Bonci E, Andreoli G, Romaggioli S, Di Miscio R, Lombardo CV, Chiesa C: Association of serum triglyceride-to-HDL cholesterol ratio with carotid artery intima-media thickness, insulin resistance and nonalcoholic fatty liver disease in children and adolescents. Nutr Metab Cardiovasc Dis 2014;24:737-743.

52 Fintini D, Chinali M, Cafiero G, Esposito C, Giordano U, Turchetta A, Pescosolido S, Pongiglione G, Nobili V: Early left ventricular abnormality/dysfunction in obese children affected by NAFLD. Nutr Metab Cardiovasc Dis 2014;24:72-74

53 Pacifico L, Di Martino M, De Merulis A, Bezzi M, Osborn JF, Catalano C, Chiesa C: Left ventricular dysfunction in obese children and adolescents with nonalcoholic fatty liver disease. Hepatology 2014;59:461-470.
Non-Alcoholic Fatty Liver and Metabolic Syndrome in Children
Horm Res Paediatr 2014;82:283-289 DOI: $10.1159 / 000365192$ 